\title{
INESTABILIDAD, COSTO DE VIDA Y SALARIOS REALES EN VENEZUELA EN EL SIGLO XIX
}

\section{INSTABILITY, COST OF LIVING, AND REAL WAGES IN VENEZUELA IN THE 19TH CENTURY}

\author{
Leticia Arroyo Abad* \\ Middlebury College, Vermont, Estados Unidos <larroyoabad@middlebury.edu>
}

Resumen. El siglo XIX fue una época tumultuosa en Venezuela. Guerras, revueltas sociales e inestabilidad política caracterizaron este periodo. Asimismo, la economía creció a tasas saludables impulsada por la expansión del sector exportador que estaba especializado en el café y el cacao. En este artículo se presentan nuevas estimaciones del costo de vida y de los estándares de vida de Caracas. La evidencia indica que el costo de vida en Venezuela, con base en estimaciones de Caracas, era alto. Más aún, los estándares de vida, medidos con el ratio de bienestar, mejoraron durante el siglo. Sin embargo, estos son bajos en comparación con otras ciudades en el mundo. Este análisis sugiere que la inestabilidad es costosa. Las condiciones internas y la alta especialización de exportación agregaron volatilidad a una situación económica frágil.

Palabras clave: precios; costo de vida; bienestar; salario real; Caracas; Venezuela.

Abstract. The 19th century was a tumultuous period in Venezuela. Wars, social unrest, and political instability plagued this country. At the same time, the economy expanded at a healthy rate fueled by the growth of the export sector that specialized in coffee and cacao. This article presents novel estimations on the cost of living and living standards for Caracas. The evidence indicates that the cost of living was high. Moreover, the standard of living, measured as the welfare ratio, improved throughout the century; however, it was low when compared to other cities in the world. The analysis suggests that instability is costly. As evidenced by the estimates from Caracas, domestic turbulent domestic conditions and lack of diversification of the export basket added volatility to the already fragile economic situation.

Key words: prices; cost of living; welfare; real wages; Caracas; Venezuela.

Fecha de recepción: agosto de 2012. Fecha de aceptación: diciembre de 2012.

* Agradezco la ayuda provista por el Archivo General de la Nación de Venezuela y la Academia de Historia en Caracas, Venezuela, y el aporte de tres dictaminadores anónimos. Se reconoce el financiamiento de la Fundación Nacional de Ciencias (National Science Foundation), beca número 343586030799817700.

Am. Lat. Hist. Econ., año 20, núm. 3, septiembre-diciembre, 2013, pp. 114-137 


\section{INTRODUCCIÓN}

$\mathrm{E}$ n la actualidad, el petróleo es la base de la economía venezolana. Siendo el quinto exportador mundial, esta mercancía representa $80 \%$ de sus exportaciones y contribuye con $50 \%$ del ingreso gubernamental. Sin embargo, la realidad económica de este país en el siglo XIX estaba muy poco relacionada con el petróleo. En efecto, poco sabemos de su evolución económica después de la independencia. El aporte de la historia económica latinoamericana es escaso en comparación con las obras existentes sobre Argentina, Brasil y México. Con base en datos de la ciudad de Caracas, este artículo revela el desenvolvimiento económico de Venezuela durante el siglo XIX utilizando fuentes primarias y secundarias de la ciudad de Caracas.

Durante la mayor parte de su vida colonial, Venezuela fue una provincia en la frontera del imperio español. Con poco contacto con el resto del imperio, su economía fue autosuficiente hasta fines del siglo XVIII. En los tiempos borbónicos ganó dinamismo mediante la obtención del monopolio real del cacao, mientras que la corona unificaba el control territorial bajo el poder de Caracas. Las guerras de la independencia diezmaron las fuentes de producción. La recuperación de la economía venezolana fue lenta y las estimaciones de estándares de vida en este artículo reflejan la ruina económica de este país. También en el siglo XIX, Venezuela se integra a los mercados internacionales mediante la exportación de su producto tradicional, el cacao, y un producto nuevo cuya introducción se debió a las vicisitudes de la guerra: el café. A diferencia de sus vecinos Colombia y Ecuador, este país desarrolló una economía exportadora más robusta. De 1830 a 1900 las exportaciones aumentaron siete veces, impulsadas por estas dos mercancías. Ambas representaron en promedio 50\% de las exportaciones, con un pico de $75 \%$ a mediados de la década de 1870 .

Según diversos índices de actividad económica, Venezuela creció a una tasa anual de $2.6 \%$ desde 1830 hasta 1900 . Sin embargo, el crecimiento económico no se tradujo necesariamente en bienestar económico para la mayoría de la población. Mis cálculos de estándares de vida indican que el bienestar económico de los venezolanos acompañó los vaivenes de la actividad económica; se recuperó luego de las guerras de la independencia, perdió terreno durante la Guerra Federal y recuperó dinamismo hasta mediados de la década de 1890, revirtiendo esta tendencia favorable hacia fines del siglo XIX.

A pesar de que el bienestar económico hacia 1900 fue muy superior al nivel registrado en 1830, en términos de consumo de subsistencia una familia tipo apenas si podía satisfacer las necesidades básicas de consumo para su hogar, ya que el costo de vida era caro, tal como lo indican las 
estimaciones para la ciudad de Caracas. Estas indican un encarecimiento promedio de la canasta de consumo durante la Guerra Federal y en la década de 1880. Estos episodios de inflación obedecen a distintas causas. Durante la Guerra Federal hubo escasez de oferta, mientras que en la década de 1880 el alza de los precios fue consecuencia del incremento de la demanda.

En la primera sección se describen las fuentes y la metodología utilizadas para el cómputo del costo de vida y el bienestar económico en Venezuela. Acto seguido se resumen las características principales del siglo XIX en términos económicos y políticos. La tercera sección presenta las estimaciones de costo de vida anuales, continuando con el cálculo del bienestar económico y el salario real. Antes de presentar las conclusiones, se incluye un análisis comparativo del bienestar económico de Venezuela con países europeos y latinoamericanos.

\section{FUENTES Y METODOLOGÍA}

La historiografía de precios y estándares de vida en Latinoamérica se encuentra rezagada respecto a la vasta cantidad de estudios en Europa y Estados Unidos. En términos de precios, los trabajos pioneros de Romano y Florescano fueron sumamente enriquecidos con el aporte de la compilación de Johnson y Tandeter. ${ }^{1}$ En ese mismo volumen, el historiador John Coastworth sostiene que todas las ramas de especialización de la historia económica están a la espera de datos de precios pues sin ellos es difícil analizar el desarrollo de las economías temporalmente. ${ }^{2}$

A pesar de la valiosa contribución de estos autores y sus seguidores, la mayor parte de la literatura se concentra en el periodo colonial. ${ }^{3}$ Las series históricas luego se retoman hacia fines del siglo XIX, continuando, en muchos casos, hasta nuestros días. El resultado es una laguna en la mayor parte del XIX, un siglo de suma importancia en la región debido a los procesos de independencia de España y el subsecuente periodo de organización nacional latinoamericano. No es sorprendente descubrir que la desarticulación del sistema colonial trajo consecuencias tangibles en términos de la conservación y archivo de información. Como consecuencia, la búsqueda y obtención de datos ha sido más dificultosa. 1990.

${ }^{1}$ Romano, “Movimiento", 1963; Florescano, Precios, 1971, y Johnson y Tandeter, Essays,

${ }^{2}$ Coatsworth, "Economic", 1990, p. 21.

${ }^{3}$ Existen unos pocos artículos relativos al siglo XIX. En el caso de México, véase Gómez-Galvarriato y Musacchio, "Nuevo", 2000, pp. 47-91. Para Argentina, Cuesta, "Precios", 2007 y 2012. Williamson, "Real", 1999, ofrece series sobre salarios reales para varios países latinoamericanos. 
Para evaluar estándares de vida, es necesario obtener datos sobre precios al consumidor, salarios y canasta de consumo. Si bien su recopilación es un desafío, es notable la ausencia de series de salarios para la mayoría de los países latinoamericanos durante la época colonial. Sin embargo, para el siglo XIX la situación es incluso más dificultosa. La disponibilidad de salarios es escasa para todos los países de Latinoamérica, con excepción de ciertos centros urbanos en México, Perú y Bolivia. ${ }^{4}$ La más reciente contribución para la época colonial es la de Arroyo, Davies y Van Zanden, quienes estiman series de bienestar económico para centros urbanos en Argentina, Bolivia, Colombia, Chile, México y Perú para periodos seleccionados.

En este artículo, fuentes primarias y secundarias han sido utilizadas para calcular no sólo el costo de vida, sino también los salarios reales para el periodo de 1830 a 1900 en Venezuela. En términos de precios, Carrillo Batalla ha publicado dos obras con precios al consumidor anuales para 43 productos de consumo. ${ }^{5}$ Las fuentes citadas incluyen diarios nacionales, como el Diario de Avisos, y el Archivo General de la Nación de Venezuela. El propio autor también calcula un índice de precios al consumidor, sin embargo, no provee las ponderaciones de los diferentes bienes incluidos. Dada esta restricción, se han estimado dos índices de precios. El primero, denominado "índice de precios de subsistencia", sigue la metodología desarrollada por Allen para algunos países de Europa y además utilizada para otros países en Asia y América Latina ${ }^{6} \mathrm{El}$ segundo, llamado "índice de precios tradicional”, emula la estimación realizada por Gómez Galvarriato para México a fines del siglo XIX y principios del XX. ${ }^{7}$

El cuadro 1 muestra los bienes incluidos en la canasta de subsistencia. De acuerdo con sus creadores, Allen et al., la elección de una canasta de subsistencia mínima obedece a distintos niveles y costos de vida en el mundo. La canasta inicial creada por Allen para Inglaterra resultaba muy onerosa para países en vías de desarrollo. Para hacer esta canasta más acorde con las realidades de estos países, los autores redujeron el consumo de los principales bienes. Comprende dos grupos de bienes en general: alimentación y otros. En el rubro de alimentación se computa una canasta de 1940 calorías y 60 gramos de proteínas diarias con una dieta de frijoles con una pequeña porción de carne suplementado con el carbohidrato de preferencia. En este caso utilizamos el maíz. Adicionalmente, en el rubro de otros se incluyen otros bienes no alimenticios tales como velas y combustible. Puesto que esta canasta especifica las cantidades de cada uno de

${ }^{4}$ Arroyo, Davies y Zanden, “Conquest”, 2012.

${ }^{5}$ Carrillo, Cuentas, 2001 y 2002.

${ }^{6}$ Allen, "Great", 2001; Allen et al., "Wages", 2011; Arroyo, Davies y Zanden, "Conquest", 2012; Özmucur y Pamuk, "Real”, 2002, y Zanden, "Wages”, 1999.

${ }^{7}$ Gómez-Galvarriato, "Evolution”, 1998. 


\section{CUADRO 1. CANASTA DE BIENES MÍNIMA: NIVEL DE SUBSISTENCIA}

\begin{tabular}{llccc}
\hline & & & & $\begin{array}{c}\text { Cantidad por } \\
\text { persona por año }\end{array}$ \\
& Unidad & Calorías & Proteínas & \\
Alimentos y bebidas & $\mathrm{kg}$ & 3370 & 70 & 165 \\
Maíz & $\mathrm{kg}$ & 1455 & 71 & 45 \\
Frijoles & $\mathrm{kg}$ & 2500 & 200 & 35 \\
Manteca & $\mathrm{kg}$ & 7286 & 7 & \\
Otros & & & & 1.3 \\
Jabón & $\mathrm{kg}$ & & & 3 \\
Textiles & $\mathrm{kg}$ & & & 1.3 \\
Velas & $\mathrm{kg}$ & & & 3 \\
Kerosene & $\mathrm{kg}$ & & & 1.3 \\
Combustible & $\mathrm{MBTU}$ & & & 60 \\
Total de calorías por persona & & & \\
Total de proteínas por persona & & & \\
& & & \\
\hline
\end{tabular}

Fuente: Arroyo, Davies y Zanden, “Conquest”, 2012, p. 153.

estos ítems, es posible calcular el costo en moneda local de la misma. El costo de vida para una familia tipo de cuatro personas se estima multiplicando el costo de la canasta por tres, ya que se asume que de una familia de cuatro, dos son menores que consumen en conjunto el equivalente a un adulto. Como se ha construido de manera consistente con la metodología de Allen et al., se podrá realizar una comparación del bienestar económico con otras ciudades en el mundo.

La segunda canasta es más tradicional ya que incluye artículos más variados - por ejemplo distintas proteínas y bebidas alcohólicas como el aguardiente. Adicionalmente, esta canasta utiliza ponderaciones relativas por tipo de bien de consumo sumando en su totalidad 100\% (véase cuadro 2). Para facilitar la comparación con la canasta de subsistencia, se han clasificado los bienes en rubros similares: alimentación y bebidas, y otros. La composición relativa es $83.5 \%$ en alimentos y bebidas y el resto en otros. A simple vista, se aprecia que la cantidad de bienes incluidos es mayor que en la canasta de subsistencia. Esta estimación está basada en presupuestos de familias de obreros en México, tal como lo cita su autora Gómez-Galvarriato. ${ }^{8}$ La consulta de fuentes secundarias ha provisto información sobre 


\section{CUADRO 2. CANASTA TRADICIONAL: VARIEDAD DE BIENES}

\section{Ponderación (porcentaje)}

$\begin{array}{lr}\text { Alimentos y bebidas } & 83.5 \\ \text { Arroz } & 2.4 \\ \text { Azúcar } & 5.2 \\ \text { Café } & 4.6 \\ \text { Carne } & 17.7 \\ \text { Frijoles } & 6.8 \\ \text { Vegetales } & 6.3 \\ \text { Manteca } & 7.8 \\ \text { Maíz } & 22.6 \\ \text { Papas } & 1.4 \\ \text { Pescado } & 1.4 \\ \text { Queso } & 1.3 \\ \text { Sal } & 1.9 \\ \text { Aguardiente } & 4.0 \\ \text { Otros } & 16.5 \\ \text { Jabón } & 1.2 \\ \text { Tabaco } & 0.8 \\ \text { Textiles } & 7.0 \\ \text { Velas } & 7.5 \\ \text { Total } & 100.0\end{array}$

Fuente: elaborado con base en Gómez-Galvarriato, "Evolution”, 1998.

el contenido de la canasta de consumo venezolana y, consecuentemente, se ha adaptado el presupuesto teniendo en consideración los patrones de consumo en Venezuela y según la disponibilidad de datos. Por ejemplo, se ha notado que la canasta de consumo popular del venezolano era primordialmente carne, lácteos y granos. En términos de bebidas alcohólicas, el aguardiente reemplaza al tradicional vino y a la cerveza, que son comúnmente utilizados en los presupuestos en los países europeos. ${ }^{9}$

En general, las series de precios son continuas, no presentan brechas. Puesto que los datos provienen de dos fuentes que cubren periodos consecutivos, no existe, necesariamente, continuidad entre todas las series de datos del primer periodo (1831-1873) y las del segundo (1874-1900). ${ }^{10}$ Para

${ }^{9}$ Cartay, Historia, 1988, pp. 131-132.

${ }^{10}$ Este hecho implica el uso de una canasta de consumo más limitada. 
compatibilizar los dos periodos, se han hecho conversiones monetarias y físicas. En principio, los precios en el primer periodo están denominados en reales y en unidades no métricas (por ejemplo la libra) mientras que en el segundo periodo se utiliza el bolívar como unidad monetaria y el sistema métrico. La conversión del sistema imperial al métrico no presentó mayores problemas, ya que existen guías disponibles. ${ }^{11}$ Consultando fuentes oficiales, se han podido convertir los reales a bolívares de acuerdo con la siguiente fórmula: ocho reales $=$ un peso $(\$)=$ un venezolano $(\mathrm{V})$ = cinco bolivares $(\mathrm{B})$. El gobierno venezolano introdujo el venezolano en 1871 y luego el bolívar en $1879 .{ }^{12}$ Debido a las brechas en algunas series, se ha adoptado la siguiente solución: se calculó el impacto promedio que dicho bien tiene en la canasta y en los casos de falta de datos se le agregó una proporción de acuerdo con la serie histórica. ${ }^{13}$ Para verificar los datos de Carrillo Batalla, se ha consultado la obra de Cartay, que proporciona precios al consumidor de un puñado de productos. ${ }^{14}$

Los salarios fueron recopilados en el Archivo General de la Nación de Venezuela. Los gobiernos de la nueva nación venezolana presentaban presupuestos anuales que detallaban los sueldos de los empleados en las distintas entidades públicas. Estas incluían importantes sectores como el Ministerio de Hacienda, el del Interior y el de Guerra, y también instituciones de servicios públicos como el Correo Nacional, hospitales y escuelas nacionales. Los salarios seleccionados corresponden a ocupaciones de baja calificación tales como porteros, sirvientes y peones. Se optó por estas ocupaciones ya que las obras descritas anteriormente utilizan categorías similares. De esta manera es posible realizar una comparación consistente del bienestar de estos segmentos de la población. Además de los presupuestos, también fue posible encontrar decretos esporádicos sobre salarios en las distintas dependencias públicas. ${ }^{15}$ Dada la naturaleza de estos documentos, los salarios corresponden a partidas anuales pertenecientes al año fiscal.

Siendo los datos salariales relativos al sector público, es necesario considerar su representatividad respecto a la fuerza laboral venezolana duran-

${ }^{11}$ Véanse por ejemplo los archivos disponibles en Global Prices and Income History Group en $<$ http://gpih.ucdavis.edu $>$.

${ }^{12}$ Decreto del 11 de mayo de 1871 sobre la acuñación de moneda.

${ }^{13}$ Por ejemplo, sólo se han encontrado datos esporádicos de textiles, en particular la lana, en el segundo periodo. En promedio, este ítem representa 1.23\% de la canasta desde 1831 hasta 1873. Para remediar esta situación, se ha agregado $1.3 \%$ extra al costo de la canasta sin textiles. La misma metodología se aplicó para el caso del queso. Esta metodología es congruente con las estimaciones realizadas para otros países.

${ }^{14}$ Cartay, "Historia", 1988, p. 132.

${ }^{15}$ Archivo General de la Nación de Venezuela, Recopilación de Leyes y Decretos de Venezuela (1830-1903). 
te este periodo. En principio todos estos datos son urbanos, en particular de la ciudad de Caracas. ${ }^{16}$ Debido a la escasez de series de tiempo de salarios rurales es imposible realizar una comparación. Para otros países, otros investigadores estiman que la razón entre salarios rurales y urbanos osciló entre 50 y $90 \% .{ }^{17} \mathrm{Al}$ ser datos de dependencias públicas es posible que no sean representativos del resto del mercado laboral caraqueño. Cartay ofrece una perspectiva que confirma la tendencia y los niveles salariales presentados en este análisis. Afirma que "las remuneraciones [...] de los trabajadores agrícolas y urbanos y de los empleados públicos de baja categoría eran muy bajas". ${ }^{18}$ Desde el punto de vista cuantitativo, el autor presenta unos pocos datos de ocupaciones varias en el sector privado para distintas fechas. La comparación con la serie construida para este estudio muestra poca discrepancia. ${ }^{19}$

Otra salvedad relevante es la referente a la presencia de esclavitud durante parte del periodo de análisis. Como tantos otros países, Venezuela utilizó mano de obra esclava. La manumisión no ocurrió inmediatamente después de la declaración de la independencia sino décadas más tarde, en $1854 .^{20}$ Sin embargo, la población esclava disminuyó considerablemente, de 87100 en 1810 a 11967 en 1854, reduciendo, en general, el impacto sobre el mercado laboral. ${ }^{21}$

\section{VENEZUELA EN EL SIGLO XIX}

La lucha por la independencia del imperio español fue sumamente costosa para Venezuela. En efecto, a diferencia de otras regiones, Venezuela fue el campo de batalla de las guerras de independencia de la República de Gran Colombia. Bolívar ilustra la situación elocuentemente en 1814: "Todo ha sido anonadado. El fuego ha reducido a cenizas las ciudades y las campiñas: el hierro ha demolido los edificios que no devoraron las llamas; el hacha del verdugo ha inmolado al niño, al anciano, a la virgen, al inválido." ${ }^{22}$

Los costos no sólo fueron económicos, sino también en términos poblacionales. Comunidades completas fueron desintegradas y en las áreas

\footnotetext{
${ }^{16}$ Lamentablemente durante el periodo de investigación en Venezuela no se han encontrado registros de salarios en localidades rurales.

${ }^{17}$ Arroyo, "Persistent", 2013.

${ }^{18}$ Cartay, Historia, 1988, p. 131.

${ }^{19}$ Por ejemplo, en 1830 un jornalero percibía 300 venezolanos por año mientras que un portero del sector público recibía 225 bolívares. El salario anual de un peón era de 960 bolívares y el del sirviente llegaba a 720 bolívares.

20 "Ley", 1854.

${ }^{21}$ Brito, "Población", 1967, pp. 351-354.

${ }^{22}$ Bolívar, [sin título], 1814, p. 1.
} 
de combate la gente emigró a destinos más seguros. El terremoto de 1812 también contribuyó al despoblamiento con la muerte de 15000 a 20000 personas. ${ }^{23}$ Según las estimaciones, en 1810 la población en el territorio venezolano ascendía a 898043 habitantes, y llegó a un mínimo de 685212 quince años más tarde, esto es, una caída de alrededor de $24 \% .{ }^{24}$ Desde el punto de vista productivo, gran número de plantaciones cacaoteras fueron destruidas: sus fundos saqueados y la tierra arrasada. Mientras que los valles de Cumaná, Caracas y Aragua fueron los más afectados, la estructura productiva de este país fue comprometida, ya que el cacao había sido el símbolo de integración comercial durante la época colonial. ${ }^{25}$

El sector agrícola venezolano fue forjado por el cultivo del café y del cacao. A pesar de ser este el producto más tradicional -basado en el monopolio colonial de este fruto-, debido a la pérdida de los árboles maduros durante las guerras de independencia hubo una sustitución a favor del café. La replantación de cacaoteros implicaba costos más onerosos comparados con el café. Por ejemplo, este cultivo demanda abundante mano de obra en su etapa inicial, ya que necesita zanjas y árboles de sombra. Adicionalmente, la primera germinación ocurre luego de 30 meses, y la primera cosecha luego de cuatro años. En contraste, el capital inicial y requisitos laborales del café no eran tan costosos. En principio, la plantación de cafetales no precisa de una etapa de germinación prolongada. Además, la primera cosecha es posible en tan sólo dos años. Esta sustitución parcial en Venezuela fue posible debido a que las condiciones climáticas y la fertilidad del suelo eran favorables. Asimismo, los procesos de cosecha eran similares: ambos requerían la recolección manual de los frutos y el secado al sol en grandes patios. ${ }^{26}$

La recuperación de la actividad económica, en especial en el sector exportador, fue lenta. En la década de 1830 el gobierno procuró fomentar el sector cafetalero de exportación mediante la eliminación de alcabalas, diezmos y otros impuestos. Luego estas medidas fueron extendidas al resto de los sectores productivos. Los resultados fueron favorables, se aceleró el ritmo de la actividad económica con un aumento de $250 \%$ en las exportaciones. Tal fue el impacto que la balanza comercial se tornó superavitaria a mediados de esta década. ${ }^{27}$ Asimismo, ya a finales de este periodo, la agricultura comenzó a expandirse a nuevas áreas. ${ }^{28}$ Este impulso fue interrumpido a principios de la década siguiente; una caída de $25 \%$ en el

${ }^{23}$ Tarver y Frederick, History, 2005, p. 50.

${ }^{24}$ Baptista, Bases, 2006, p. 28, y Brito, "Población", 1967, pp. 347-348.

${ }^{25}$ Brito, Historia, 2002, e Izard, Política, 1976, p. 19.

${ }^{26}$ Barral, Avenir, 1881.

${ }^{27}$ Cálculos con base en estadísticas obtenidas de Baptista, Bases, 2006, y Veloz, Economía, 1989.

${ }^{28}$ Carrillo, Cuentas, 2001, p. 1420. 
precio internacional del café y la frágil situación financiera se tradujeron en una caída de las exportaciones cercana a $27 \%$ en términos constantes.

El rumbo de la economía venezolana desde 1840 hasta mediados de la década de 1860 se vio influido por la gran inestabilidad política y social. En vista de las numerosas revueltas sociales, el gobierno expandió su fuerza militar, engendrando crecientes presiones fiscales. A pesar de la favorable balanza comercial en este periodo, la corrupción y el caos gubernamentales dejaron al país en una situación fiscal muy precaria. La liberalización de los esclavos en 1854 fue también un factor desestabilizante, ya que el gobierno no pudo pagar la compensación a los hacendados. La caída de los precios de exportación hacia fines del periodo empeoró aún más la delicada posición del país. Con una recaudación fiscal en caída, la administración no pagaba los sueldos de los empleados públicos, y otorgaba vales que se cotizaban entre 5 y $10 \%$ de su valor original. ${ }^{29}$ La presión social y política llegó a tal magnitud que el país se sumergió en un periodo de hostilidad civil llamado la Guerra Federal. Durante estos largos cinco años (1859-1864) Venezuela perdió alrededor de 100000 vidas, y la actividad económica disminuyó agudamente.

No fue sino hasta 1870 que Venezuela logró estabilidad política. El gobierno central consiguió controlar poco a poco la totalidad de la nación. Mediante un pacto con los caudillos regionales, se logró disminuir los niveles de violencia y a su vez reducir los gastos militares. A fin de integrar el territorio nacional, el gobierno impulsó un programa de obras públicas que llegó a abolir un sinfín de peajes internos locales. Si bien el ritmo de la actividad económica alcanzó niveles nunca vistos en Venezuela, nuevamente la caída de los precios de exportación del café a fines de la década de 1870, junto con una mala cosecha por la plaga de la langosta, deprimieron las exportaciones y la producción. Sin embargo la estabilidad política atrajo capitales extranjeros que financiaron el desarrollo de los centros urbanos venezolanos, especialmente Caracas, transformándola en una capital moderna. ${ }^{30}$

La última década del siglo está caracterizada por inestabilidad política ante un trasfondo económico desfavorable. Los vaivenes en la actividad económica fueron mucho más frecuentes que en las décadas anteriores. En 1900 el nivel de la producción fue menor que a principios de la década. Las exportaciones, la base de la economía venezolana, presentaron una tendencia descendente con precios en niveles bajísimos. Para 1900 los

\footnotetext{
${ }^{29}$ Matthews, “Turbulenta”, 1976, pp. 106-116.

${ }^{30}$ Frankel, "Política", 1976, pp. 173, 194 y 197.
} 
precios del café y del cacao disminuyeron 60.4 y $26.5 \%$, respectivamente. En el caso del café, el precio más bajo del siglo fue registrado en ese año. ${ }^{31}$

En general, Venezuela en el siglo XIX estuvo afligida por la inestabilidad política y económica, con breves respiros de estabilización. SalcedoBastardo sostiene que desde 1830 hasta 1935 el país solamente vivió cortos periodos pacíficos: 1839-1840, 1842-1843, 1924-1927, y 1932-1835. ${ }^{32} \mathrm{El}$ desarrollo de la economía exportadora siguió el ritmo de los precios internacionales del café y del cacao, dada la ausencia de la diversificación en la canasta de bienes de exportación. A pesar de la volatilidad en ambos flancos, política y economía, este país creció $2.5 \%$ anual desde 1830 hasta $1900 .{ }^{33}$ Empezando con una economía en las ruinas después de las guerras de independencia, Venezuela creció a pesar de una guerra interna de cinco años, con precios de exportación desfavorables y una coyuntura política caótica en ciertos puntos. Esta volatilidad persistente caracterizó a la economía venezolana antes del surgimiento del controversial sector petrolero.

\section{EL COSTO DE VIDA}

Los precios al consumidor en Venezuela durante el siglo XIX presentaron oscilaciones anuales importantes. ${ }^{34}$ La volatilidad, medida como la desviación estándar de las variaciones relativas anuales, es de 0.16 y 0.09 para los índices de precios de la canasta de subsistencia y tradicional respectivamente. En la gráfica 1 se presentan las dos series de costo de vida. Una inspección informal de las mismas revela distintas tendencias en este siglo. Más formalmente, es posible calcular quiebres de tendencia en estas series utilizando métodos econométricos tales como el cálculo recursivo de parámetros en estimaciones de series de tiempo. De esta manera, se evalúa la estabilidad de dichos parámetros en el tiempo, obteniendo endógenamente las fechas de los quiebres.

Para estimar estos quiebres de tendencia se empleó un sistema autorregresivo de tipo $\mathrm{AR}(3)$. La forma funcional de esta estimación es, para

${ }^{31}$ Cálculos con base en Baptista, Bases, 2006, y Veloz, Economía, 1989.

${ }^{32}$ Salcedo-Bastardo, Historia, 2004, p. 337. Existieron, según el autor, otros periodos de tranquilidad relativa tales como 1850, 1852, 1876, 1890, 1891, 1893-1894, 1897, 1904, 1906, 1909-1910.

${ }^{33} \mathrm{Ibid}$.

${ }^{34}$ Cabe notar que Baptista, Bases, 2006, presenta un índice de costo de vida para este periodo. La correlación con el índice de subsistencia y con la canasta básica es de 0.10 y -0.04 respectivamente. No lo presentamos aquí ya que en su estimación sólo se utilizan los índices de precios de exportación y de importación. El problema con esta metodología radica en que a) las exportaciones e importaciones estaban concentradas en un puñado de bienes y b) los precios son al por mayor. Dadas estas objeciones, es razonable concluir que este índice no refleja fielmente el costo de vida del consumidor venezolano. 


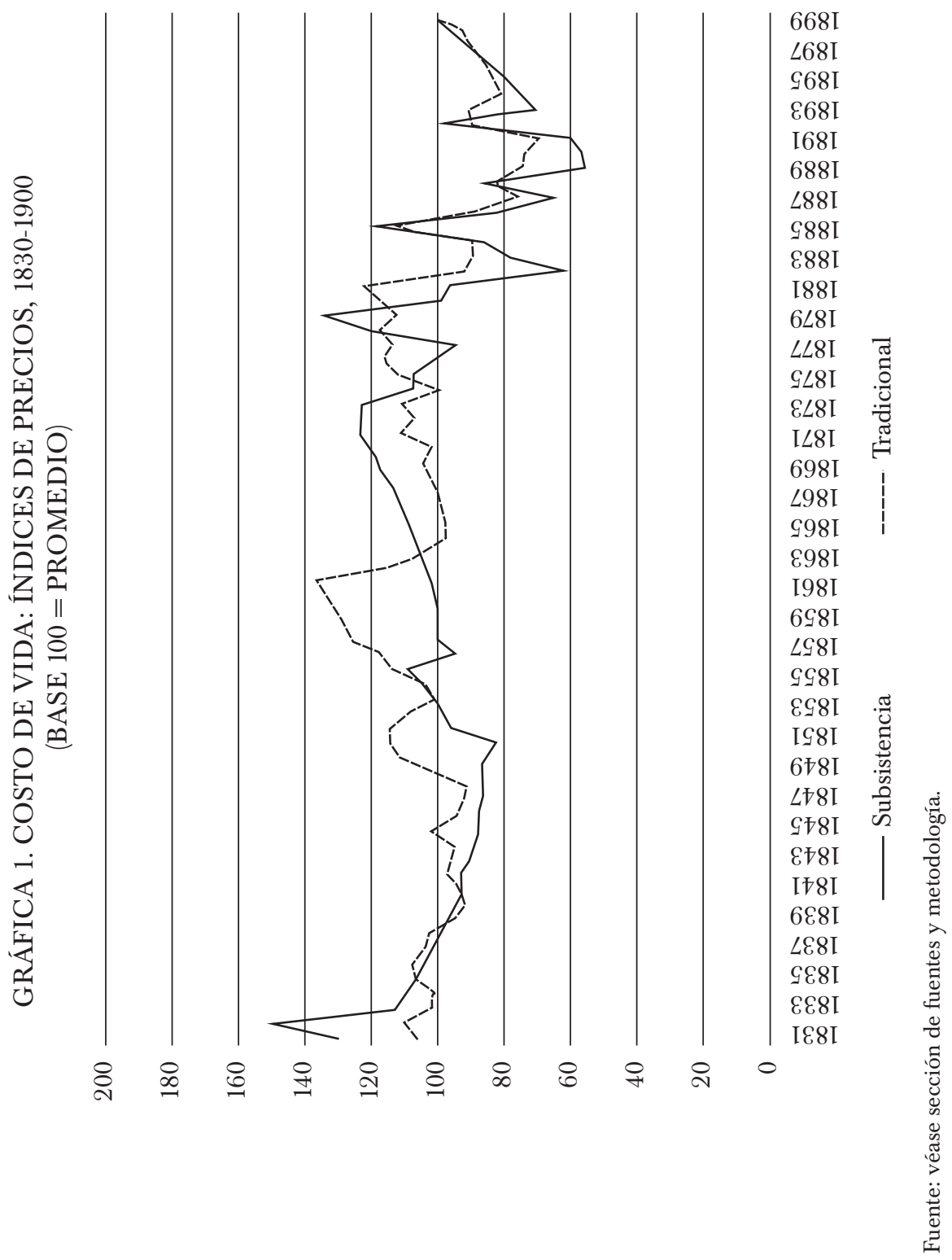


ser específica, $P_{t}=\alpha+\beta_{1} P_{t-1}+\beta_{2} P_{t-2}+\beta_{3} P_{t-3}+\varepsilon_{t}$, en donde $P_{t}$ es el índice de precios en el periodo $t, P_{t-1}$ es el índice de precios en el periodo $t-1$ y así sucesivamente y $\varepsilon_{t}$ es un término de error. Mediante la estimación recursiva de esta ecuación es posible obtener los coeficientes $\alpha, \beta_{1}, \beta_{2}, \mathrm{y} \beta_{3}$. Dada la volatilidad de las series es razonable que los coeficientes no sean sumamente estables. Sin embargo, es posible identificar un periodo en particular en que los parámetros varían significativamente. En principio, desde fines de 1850 hasta mediados de 1860, los parámetros son inestables y sólo se normalizan hacia 1870. Este comportamiento de los parámetros es común a ambos índices de costo de vida. La gráfica 2 presenta los coeficientes en esta estimación recursiva con una ventana de 20 periodos, incluyendo zonas sombreadas en las que los coeficientes presentan cambios considerables.

Volviendo a la gráfica 1, antes de la Guerra Federal el índice de subsistencia descendió en promedio. Este comportamiento se explica por el descenso de precios de dos artículos de consumo importantes en la dieta de los venezolanos: el maíz y los frijoles. Ambos presentan tendencias descendentes en este periodo. Por otra parte, el incremento de precios en bienes de consumo menos importantes en la canasta tales como el jabón y las velas no logran revertir esta tendencia descendente. Utilizando promedios quinquenales, el costo de vida descendió $20.7 \%$ en esta etapa. En la canasta tradicional, la caída de precios en general es más módica, llegando tan sólo a 3.3\% -utilizando también promedios quinquenales. Este comportamiento se debe a incrementos en bienes incluidos en la canasta tradicional, en particular el tabaco y el aguardiente. Asimismo, puesto que la canasta es más variada, la disminución de los precios de los bienes primordiales de consumo no tuvo tanto peso en el índice en general.

No es de sorprender el incremento de los precios en general durante el periodo de guerra civil. Ambos índices muestran una suba importante. La canasta de subsistencia subió 11\%, ya que sólo los precios de la carne y las velas aumentaron de manera apreciable. En el caso del índice tradicional, $70 \%$ de los bienes experimentó alzas considerables, el resto se mantuvo estable o con poca variación. El resultado neto fue un alza de 34\% hasta 1862 y una tendencia descendente hacia finales del periodo.

La relativa consolidación política obtenida en la década de 1870 se tradujo a su vez en un costo de vida con una ligera tendencia descendente. Esta tendencia fue revertida hacia el fin del siglo. Dada la severidad de las condiciones económicas y políticas de fines de siglo, no es sorprendente observar esta tendencia a la alza. En particular, la carne, el aguardiente, el jabón y los textiles experimentaron incrementos en sus precios, mientras que los del azúcar, el queso, los frijoles y los vegetales se mantuvieron sin mayores cambios. 


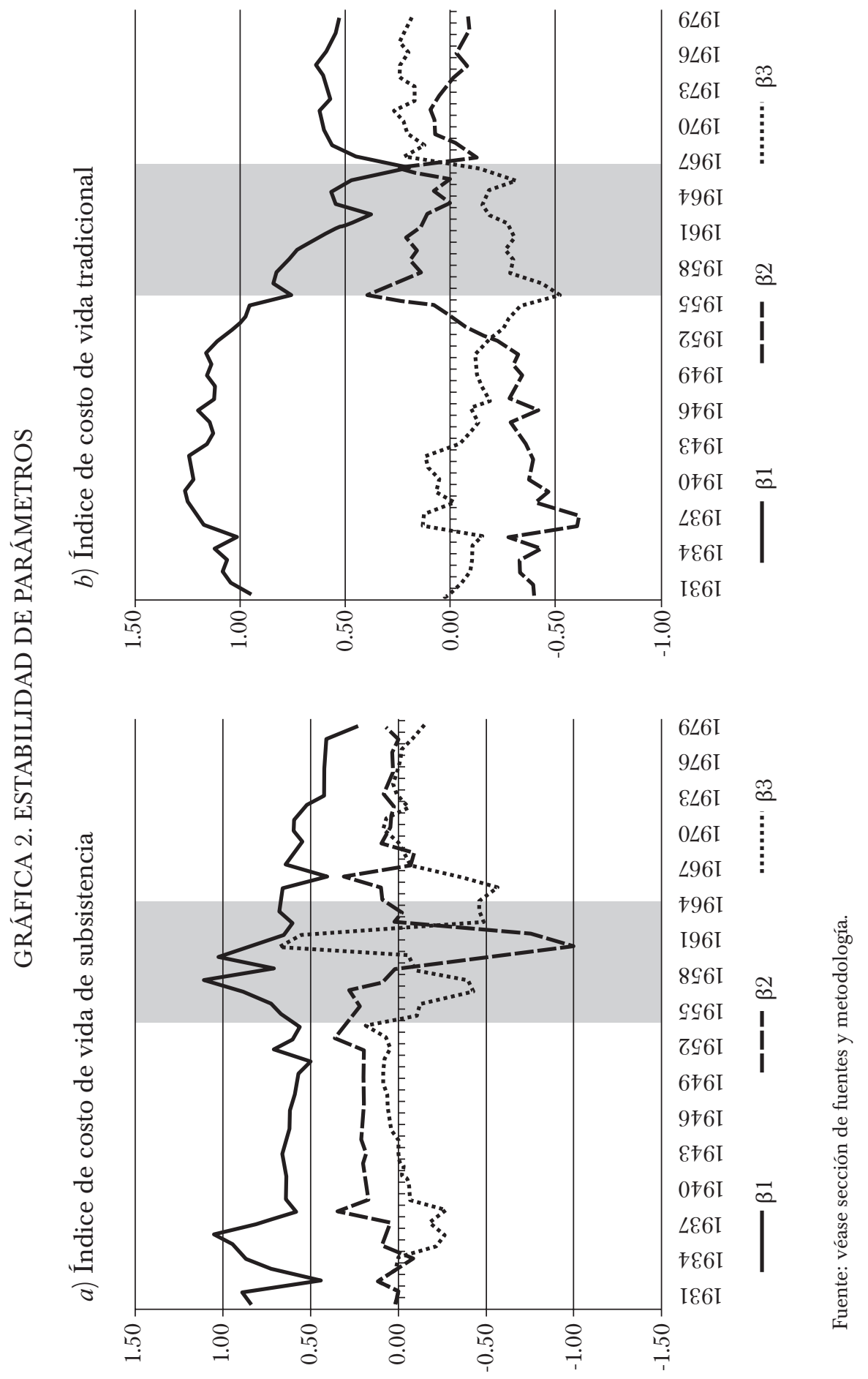




\section{SALARIO REAL Y LA EVOLUCIÓN DE LA ACTIVIDAD ECONÓMICA}

Si bien el índice de precios nos permite delinear la evolución del costo de vida, no es posible determinar con certeza el bienestar de la población sin datos de salarios. Tal como se explicó en la sección de fuentes y metodología, se ha optado por el uso del salario del trabajador no calificado en la ciudad de Caracas. En términos nominales los salarios aumentaron considerablemente hasta la década de 1850 para mantenerse sin cambios considerables por el resto del siglo.

El cómputo de dos índices de costo de vida es particularmente informativo en la evaluación de la evolución del bienestar venezolano en el siglo XIX. Usando la canasta de subsistencia es posible determinar si una unidad familiar podía satisfacer las necesidades básicas. Técnicamente, se estima este ratio como el cociente entre el salario nominal y la canasta de subsistencia multiplicada por tres para tener en cuenta el consumo de toda la familia. Este indicador posee una interpretación específica, ya que permite determinar el poder adquisitivo del salario nominal respecto a un nivel de consumo mínimo, de subsistencia. Con un ratio de uno, el poder adquisitivo del salario es exactamente igual al costo del nivel de consumo de subsistencia. Un cociente mayor a uno implica un salario mayor a lo necesario para satisfacer el consumo mínimo. Consecuentemente, un cociente menor a uno indica que con ese nivel de salario nominal, una familia no puede adquirir la mínima cantidad de productos para subsistir.

La gráfica 3 presenta el promedio quinquenal del ratio de bienestar. Es claro que a comienzos del periodo, el nivel de bienestar estaba muy por debajo del nivel de subsistencia. Venezuela comienza su vida como nación independiente con un nivel de vida paupérrimo. Estas estimaciones confirman entonces las observaciones cualitativas del periodo, las guerras de la independencia dejaron una huella muy costosa en términos económicos. Sin embargo, la relativa estabilidad ganada en las sucesivas décadas se traduce en una reducción del costo de vida conjuntamente con un incremento en los salarios nominales. Como resultado, el ratio de bienestar aumenta hasta los albores de la Guerra Federal, cuando este llega a casi 0.90. El deterioro del bienestar económico es continuo hasta fines de la década de 1860, en donde se observa el punto de inflexión.

Tal como se ha comentado en la sección de contexto histórico, en las décadas de 1870 y 1880 se logró mayor estabilidad política y junto con la entrada de capitales del exterior se impulsó la actividad económica, en particular por parte del gobierno. Es durante estas décadas que se observa un incremento sustancial del bienestar que trepa a niveles superiores a 1.2 en la primera mitad de la década de 1890. Esta tendencia alentadora es 


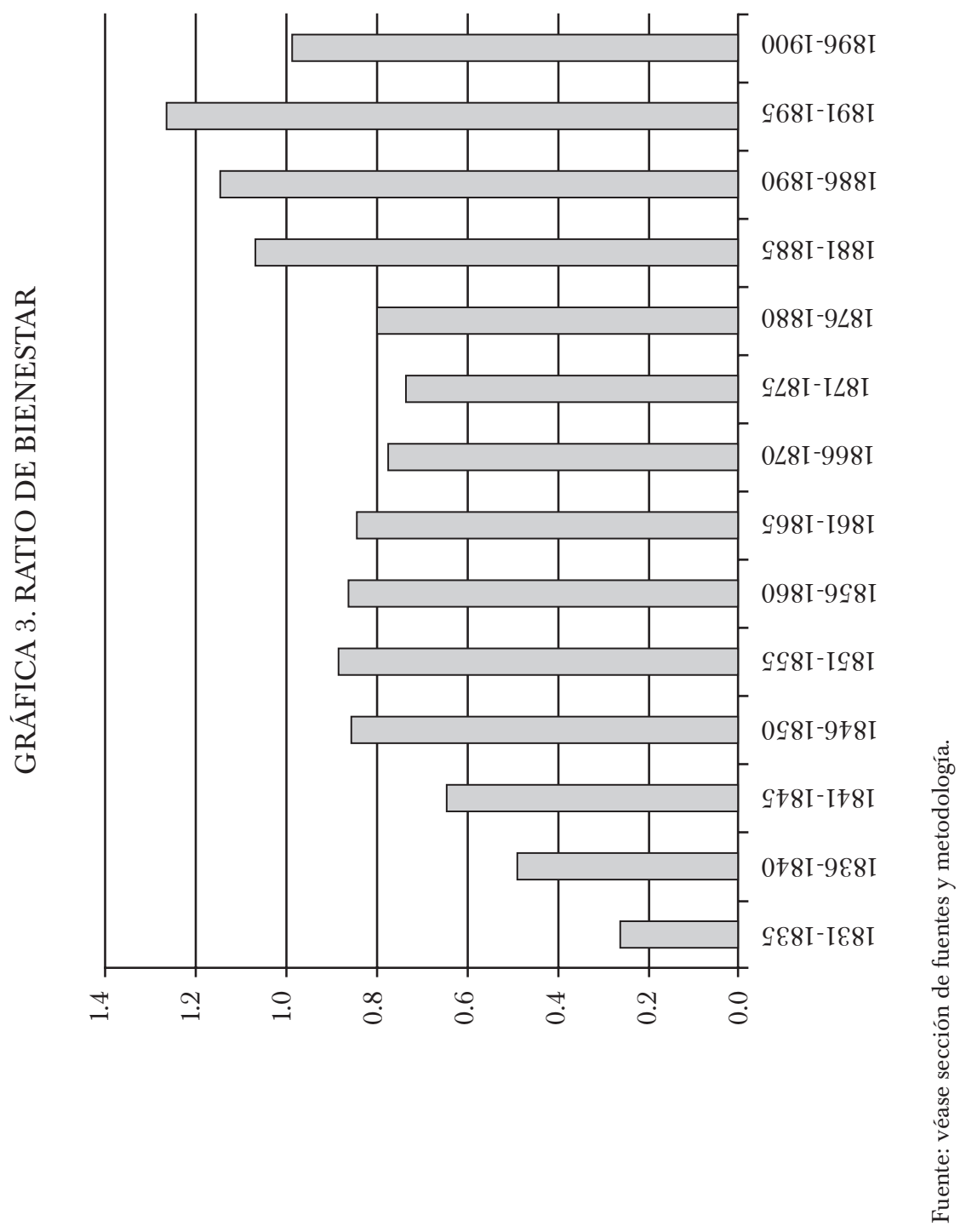


interrumpida a fines de siglo con una caída a niveles de subsistencia. Esta reversión se debe a un aumento del costo de vida de subsistencia.

Con el otro índice de costo de vida, el denominado tradicional, se ha calculado un índice de salario real a precios de 1900. La tendencia es similar a la observada en la serie de ratio de bienestar (véase gráfica 4). En efecto, el coeficiente de correlación de ambas series es de 0.7. La evolución de los salarios reales presenta un comportamiento similar a la experimentada por el ratio de bienestar. En principio se observa una expansión importante del salario real desde 1830 hasta la Guerra Federal. En efecto, el incremento fue de $164.6 \%$. Parte de esta importante alza es una recuperación del poder adquisitivo posguerra de la independencia. La guerra civil con su efecto en el aumento en el costo de vida cercenó el terreno ganado en las décadas anteriores. En salario real perdió casi un cuarto del nivel máximo preguerra alcanzado en 1854. La recuperación se aceleró hacia fines de la década de 1860, aunque con una merma en los salarios reales en la década siguiente.

Las últimas dos décadas del siglo XIX muestran un aumento significativo de los salarios reales con una reversión hacia fines de siglo. Hasta 1888, la tendencia, en promedio, fue favorable; el aumento fue de $60 \%$ cuando se calcula desde el punto mínimo en 1882 al pico en 1890 . El resto del siglo muestra oscilaciones considerables año con año, un comportamiento esperable dada la situación política reinante.

\section{EL BIENESTAR VENEZOLANO EN PERSPECTIVA COMPARADA}

En 1883, un viajero describe en su diario de viaje a Caracas que: "Entre las clases trabajadoras se nota mucha miseria, y dificultades para ganar la vida." 35 Sin embargo, la sección previa señala que el estándar de vida había mejorado considerablemente en esa década. Para evaluar la posición económica de Venezuela, es conveniente comparar su bienestar económico con otras economías en el mundo.

Gracias al arduo trabajo de historiadores económicos es posible realizar estas comparaciones, con ciertas limitaciones. Lo óptimo sería comparar con otras economías latinoamericanas en el mismo periodo. Sin embargo, los datos para el siglo XIX son escasos en general. No obstante, es posible compararlos con los de ciertas localidades en las décadas de 1810 y 1820 .

El cuadro 3 presenta estas estimaciones para 18 ciudades en el mundo: seis en Latinoamérica, tres en Asia y el resto en Europa. A primera vista,

${ }^{35}$ Laverde, Viaje, 1885, p. 39. 


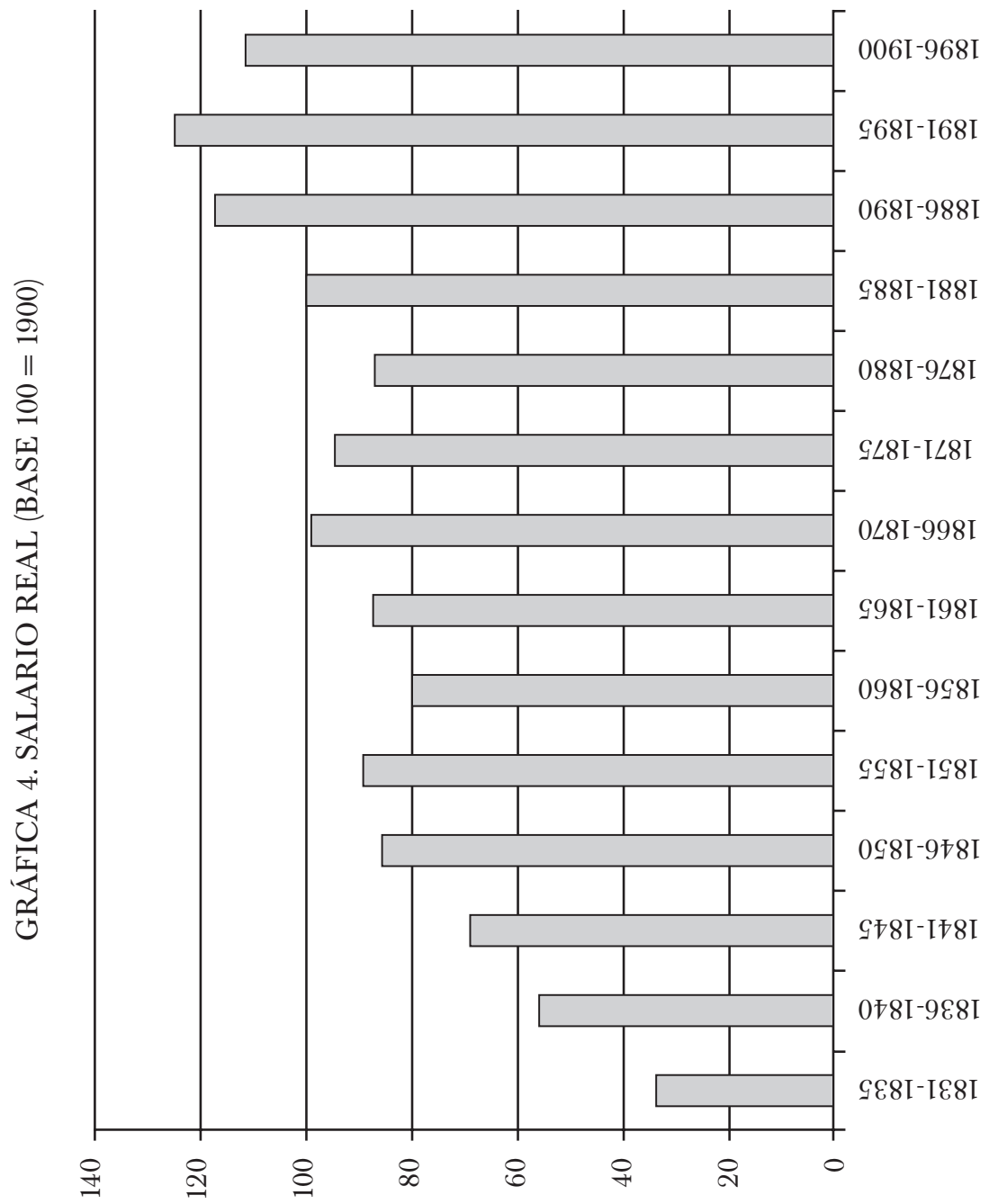


CUADRO 3. RATIOS DE BIENESTAR A PRINCIPIOS

DEL SIGLO XIX EN CIUDADES SELECCIONADAS

\begin{tabular}{lll}
\hline & Periodo & Ratio \\
Buenos Aires & 1830 & 5.22 \\
Londres & 1830 & 2.52 \\
Amberes & 1830 & 2.23 \\
Ámsterdam & 1830 & 1.97 \\
Varsovia & 1830 & 1.80 \\
Potosí & 1810 & 1.65 \\
Madrid & 1830 & 1.45 \\
Bogotá & 1800 & 1.45 \\
Estrasburgo & 1830 & 1.31 \\
Gdansk & 1810 & 1.23 \\
Cracovia & 1830 & 1.03 \\
Lima & 1820 & 0.95 \\
México & 1810 & 0.93 \\
Pekín & 1830 & 0.82 \\
India & 1830 & 0.76 \\
Milán & 1830 & 0.75 \\
Kyoto/Tokyo & 1830 & 0.69 \\
Caracas & 1830 & 0.34 \\
\hline
\end{tabular}

Fuentes: para Latinoamérica véase Arroyo, Davies y Zanden, "Conquest", 2012; para Europa, Allen, "Great”, 2001; para India, Allen, "India”, 2007, y para China y Japón, Allen et al., "Wages", 2011.

el ratio de bienestar es sumamente bajo en Caracas. Esto se debe a que se utilizó la primera década disponible, la cual coincide con una situación económica paupérrima debido a las guerras de independencia. Valores similares se observan a principios de siglo en Milán. Si se empleara el ratio correspondiente a la década previa a la Guerra Federal, el estándar de vida sería superior al de Milán y apenas inferior al de México. La comparación con China y Japón ofrece más perspectiva, los niveles de bienestar a principios del siglo XIX eran bajos comparados con Europa. En particular la serie de Pekín presenta ratios de bienestar menores al nivel de subsistencia hasta la década de 1870. Más aún, estos ratios oscilan alrededor de 0.38 hacia fines de 1850.

En perspectiva histórica, la mejora del ratio de bienestar venezolano es notable. La gráfica 5 presenta los ratios de bienestar quinquenales para 


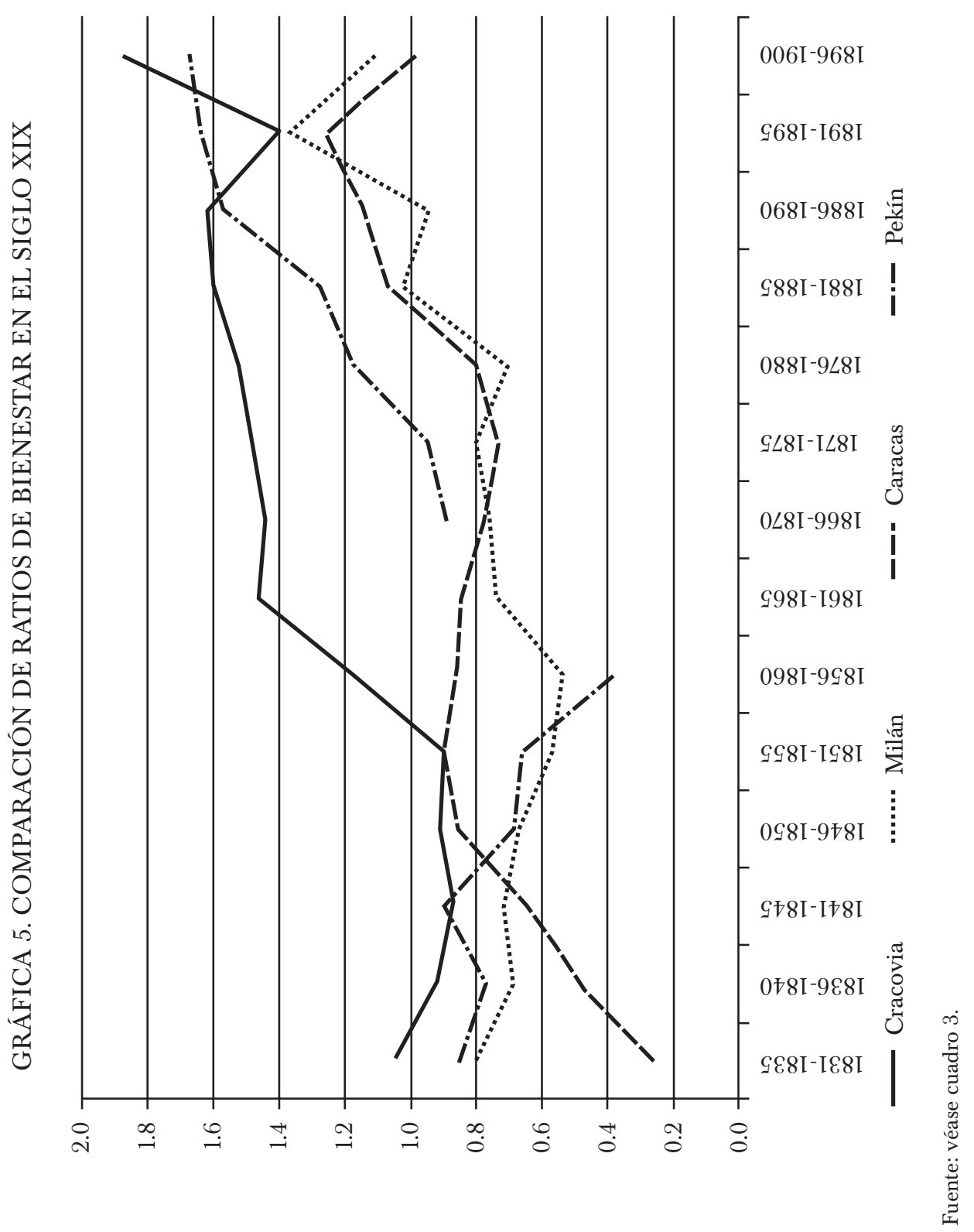


Caracas, Pekín, Cracovia y Milán. Se observa que si bien el desempeño es favorable para Venezuela, sólo Milán queda rezagado en la mayor parte del periodo. A pesar del desfavorable movimiento del ratio de Pekín a fines de 1850, dos décadas más tarde supera el estándar de vida en Caracas, incluso excediendo el nivel de subsistencia una década más tarde. El estancamiento del ratio cracoviano hasta mediados de la década de 1850 es superado en tal magnitud que una década más tarde los niveles de vida de Cracovia son de más de 40\% mayores que el nivel de subsistencia.

En resumen, la experiencia venezolana, vista a la luz de los ratios de subsistencia, es una historia de progreso respecto a su propia economía, pero de poco dinamismo cuando se compara con otras economías en el mundo. Las secuelas de las guerras de independencia, la Guerra Federal y la inestabilidad política frente a un mercado internacional volátil no permitieron que Venezuela pudiera alcanzar estándares de vida comparables con otras localidades en vías de desarrollo. La evidencia cualitativa confirma estos cálculos. Por ejemplo, Cartay observa que: "[los] salarios eran ruines y mostraban un escaso poder adquisitivo en relación con los artículos principales de la dieta popular. La pobreza de la mayoría del pueblo fue el signo dominante del siglo". ${ }^{36}$ Dadas esas condiciones, se estimaba que la esperanza de vida no superaba los 30 años en 1880. La carestía era sorprendente para los oficiales internacionales que residían en Caracas. De ellos es posible comparar el costo de diversos bienes y servicios con otras capitales latinoamericanas. Caracas resulta la más cara en 1842, situación que prevalece en los escritos hacia fines de siglo. ${ }^{37}$

\section{CONCLUSIONES}

Para Venezuela el siglo XIX fue una época de inestabilidad con moderado desarrollo económico. A pesar de un crecimiento de la actividad económica que promedió 2.6\%, los estándares de vida superaron el nivel de subsistencia tan sólo en unas pocas décadas. En este siglo que ha recibido relativamente poca atención académica, los vaivenes de la economía venezolana antes del petróleo también estuvieron influidos por el ritmo del mercado internacional.

En efecto, con una canasta de exportaciones poco diversificada, Venezuela dependía del comportamiento de dos precios de exportación clave: el del cacao y el del café. El cacao fue el legado de la economía colonial de fines del siglo XVIII, mientras que el café fue el producto de las nuevas

${ }^{36}$ Cartay, Historia, 1988, p. 135.

${ }^{37}$ Ibid., p. 138. 
condiciones posteriores a las guerras de independencia. A pesar de la expansión de la actividad agrícola, el costo de vida en Caracas fue sumamente alto. Las fuentes cualitativas confirman las estimaciones presentadas en este artículo. En términos de estándares de vida, se observa una tendencia ascendente durante el periodo con reversiones de tendencia durante la Guerra Federal y hacia fines de siglo.

Utilizando datos primarios de fuentes archivísticas para salarios y fuentes secundarias para precios, se ha estimado la evolución del costo de vida y los estándares de vida para Caracas. Dada la falta de estudios históricos de bienestar y costo de vida sobre Venezuela, este artículo ofrece nuevas estimaciones utilizando una base de datos limitada.

Estas nuevas estimaciones indican el gran costo de las guerras de independencia en Venezuela. Los estándares de vida eran sumamente bajos, evidencia de la catastrófica situación económica en este país al comienzo de su vida independiente. Como se ha adoptado la metodología creada por Allen et al. en sus numerosos artículos, se ha logrado comparar el bienestar en Caracas, Venezuela, con otras ciudades en el mundo.

De esta comparación se descubre que si bien el progreso durante el siglo XIX fue significativo, los niveles de vida, en general, fueron inferiores a los niveles de otras economías en vías de desarrollo. Esta experiencia sugiere que los costos de la inestabilidad son altos, especialmente cuando el país enfrenta una gran volatilidad doméstica y en los mercados internacionales.

\section{BIBLIOGRAFÍA}

Allen, Robert C., "The Great Divergence in European Wages and Prices from the Middle Ages to the First World War", Explorations in Economic History, Elsevier, vol. 38, núm. 4, octubre de 2001, pp. 411-447. "India in the Great Divergence" en Timothy Hatton, Kevin O'Rourke y Alan TaYlor (eds.), The New Comparative Economic History, Cambridge, Massachusetts Institute of Technology Press, 2007.

et al., "Wages, Prices, and Living Standards in China, 1738-1925: In Comparison with Europe, Japan, and India", Economic History Reviere, Economic History Society, vol. 64, núm. 1, suplemento, febrero de 2011, pp. 8-38.

Arroyo Abad, Leticia, "Persistent Inequality? The Role of Factor Endowments and Trade in Republican Latin America", Journal of Economic History, Economic History Association, vol. 73, núm 1, 2013, pp. 38-78.

Elwyn Davies y Jan Luiten van Zanden, "Between Conquest and Independence: Real Wages and Demographic Change in Spanish America, 1530- 
1820”, Explorations in Economic History, Elsevier, vol. 49, núm. 2, abril de 2012, pp. 149-166.

BAPTISTA, Asdrúbal, Bases cuantitativas de la economía venezolana, 1830-2002, Caracas, Fundación Empresas Polar, 2006.

Barral, Jean A., Avenir de Grandes Exploitations Agricoles Établies sur les Côtes du Venezuela, París, G. Masson Editeur, 1881.

Bolívar, Simón, [sin título], Gaceta de Caracas, núm. 60, 21 de abril de 1814.

Brito FigueroA, Federico, "La población y la estructura social de Venezuela en las primeras décadas del siglo XIX”, Bulletin Hispanique, Université Michel de Montaigne Bordeaux 3, vol. 69, núms. 3-4, 1967, pp. 347-364.

, Historia económica y social de Venezuela, Caracas, Universidad Central de Venezuela, 2002.

Carrillo Batalla, Tomás E., Cuentas nacionales de Venezuela, 1831-1873, Caracas, Banco Central de Venezuela, 2001. Cuentas nacionales de Venezuela, 1874-1914, Caracas, Banco Central de Venezuela, 2002.

Cartay, Rafael, Historia económica de Venezuela, Caracas, Vadell Hermanos Editores, 1988.

Coatsworth, John H., "Economic History and the History of Prices in Colonial Latin America" en Lyman L. Johnson y EnriQue Tandeter (eds.), Essays on the Price History of Eighteenth-Century Latin America, Albuquerque, University of New Mexico Press, 1990.

Cuesta, Martín, "Precios y mercados en Buenos Aires en el siglo XVIII", América Latina en la Historia Económica, Instituto de Investigaciones Dr. José María Luis Mora, vol. 14, núm. 2, julio-diciembre de 2007, pp. 25-57.

, "Precios y salarios en Buenos Aires durante la Gran Expansión (18501914)", Revista de Instituciones, Ideas y Mercados, Instituto Universitario ESEADE, vol. 56, mayo de 2012, pp. 159-179.

Florescano, EnRIQue, Precios del maízy crisis agrícolas en México, 1708-1810, México, El Colegio de México, 1971.

Frankel, Benjamín A., "Política y economía en los tiempos de Guzmán Blanco: centralización y desarrollo" en Miguel IzARD et al., Política y economía en Venezuela, 1810-1976, Caracas, Fundación John Boulton, 1976.

Gómez-Galvarriato, Aurora, "The Evolution of Prices and Real Wages in Mexico from the Porfiriato to the Revolution" en John H. Coatsworth y Alan M. TAYLOR (ed.), Latin America and the World Economy, Cambridge, Harvard University Press, 1998.

y Aldo Musacchio, "Un nuevo índice de precios para México, 18861929”, El Trimestre Económico, Fondo de Cultura Económica, núm. 265, eneromarzo de 2000, pp. 47-92.

Izard, Miguel et al., Política y economía en Venezuela, 1810-1976, Caracas, Fundación John Boulton, 1976. 
Johnson, Lyman L. y Enrique TAndeter (eds.), Essays on the Price History of EighteenthCentury Latin America, Albuquerque, University of New Mexico Press, 1990.

Laverde Amaya, Isidoro, Un viaje a Caracas, Bogotá, Tipografía de Ignacio Borda, 1885.

"Ley declarando abolida para siempre la esclavitud en Venezuela", 24 de marzo de 1854 por el Senado y Cámara de Representantes de la República de Venezuela reunidos en Congreso.

Matthews, Robert P., "La turbulenta década de los Monangas, 1847-1858" en MiGUel IZARD et al., Política y economía en Venezuela, 1810-1976, Caracas, Fundación John Boulton, 1976, pp. 106-116.

Moraes, María Inés, "Las fuentes hacendísticas para el estudio de los precios y salarios de Montevideo colonial”, Informe auxiliar núm. 3, Proyecto de investigación Caracterización sociodemográfica y económica de las sociedades preindustriales de la Cuenca del Río de la Plata, Comisión Sectorial de Investigación Científica de la Universidad de la República, 2011.

Özmucur, Suleyman y ŞEvket Pamuk, "Real Wages and Standards of Living in the Ottoman Empire, 1489-1914", Journal of Economic History, Economic History Association, vol. 62, núm. 2, 2002, pp. 293-321.

Romano, Ruggiero, "Movimiento de precios y desarrollo económico: el caso de Sudamérica en el siglo XVIII”, Desarrollo Económico, Instituto de Desarrollo Económico y Social, vol. 3, núms. 1-2, 1963.

Salcedo-Bastardo, José Luis, Historia fundamental de Venezuela, Caracas, Ediciones de la Biblioteca, 2004.

Tarver, H. Michael y Julia C. Frederick, The History of Venezuela, Westport, Greenwood Press, 2005.

Veloz, RAmón, Economía y finanzas de Venezuela, 1830-1944, Caracas, Italgráfica, 1989. Williamson, JefFrey, "Real Wages, Inequality, and Globalization in Latin America Before 1940", Revista de Historia Económica, Universidad Carlos III/Centro de Estudios Políticos y Constitucionales, año 17, núm. extra 1,1999, pp. 101-142.

Zanden, Jan Luiten VAN, "Wages and the standard of living in Europe, 1500-1800”, European Review of Economic History, European Historical Economics Society, núm. 3, vol. 2, 1999, pp. 175-198. 